\title{
The roles of Emojis in Mobile Phone Notifications
}

\author{
Channary Tauch \\ Computer \&Technology \\ Nottingham Trent University \\ Channary.tauch@ntu.ac.uk \\ Eiman Kanjo \\ Computer \&Technology \\ Nottingham Trent University \\ Eiman.kanjo@ntu.ac.uk
}

Permission to make digital or hard copies of all or part of this work for personal or classroom use is granted without fee provided that copies are not made or distributed for profit or commercial advantage and that copies bear this notice and the full citation on the first page. Copyrights for components of this work owned by others than ACM must be honored. Abstracting with credit is permitted. To copy otherwise, or republish, to post on servers or to redistribute to lists, requires prior specific permission and/or a fee. Request permissions from

Permissions@acm.org.

Ubicomp/ISWC'16 Adjunct, September 12-16, 2016, Heidelberg, Germany

2016 ACM. ISBN 978-1-4503-4462-3/16/09 \$ \$15.00

DOI: http://dx.doi.org/10.1145/2968219.2968549

\begin{abstract}
The texts in mobile messages are not always easy to decipher since tone and body language is removed from the context. Emojis offer an attractive way to express emotions to avoid misunderstandings of message tone. In this paper we shed the light on the roles of Emojis in phone notification, we conducted an in-situ study to gather phone notification data. We outline the relationship between Emojis and various social network applications including WhatsApp, Facebook and Twitter. Early results allow us to draw several conclusions in relation to number, position, type and sentimental value of Emojis. It turns out that most popular Emojis in one social app is not as popular in the others. Emojis sentimental polarity in Twitter is high and overall number of Emojis is less than Facebook. The sentimental value of Emojis is more meaningful when there are multiple Emoji in one notification.
\end{abstract}

\section{Author Keywords}

Phone Notifications; Pervasive Computing; Sentimental Analysis; Emojis; Affective Computing.

\section{ACM Classification Keywords}

H.1.2 [Information Systems] User/Machine Systems Human Information Processing 


\section{Introduction}

The advancement of computer and communication technologies has changed the use of mobile phone tremendously. Mobile phones are flooded with

notifications; competing for users' attention ranging from incoming social messages, availability of Wi-Fi networks or updates from health applications. Socia notifications, in particular are of a great value to the users; however, text messages cannot express emotion in similar fashion to face-to-face communication. This is why many people resort to use visual representation to articulate their emotions, by embedding Emojis in their messages.Emojis enable people to be more expressive in conveying their feelings and moods via a text-based communication.

On the other hand people use have different preferences for social media applications. WhatsApp, Twitter and Facebook are some of the most popular social media applications see Figure 1.

Many previous research projects have looked at Emojis usage and its sentimental values in Twitter, but no other studies have looked at Emojis in phone notifications across various social applications and how users use them and react to them in everyday interaction. In order to bridge this gap, in this work we conduct an in-situ study to mobile notifications from 12 users over 5 weeks using a mobile phone application called 'NotiMind'. In this paper we give a background and brief review of research on Emojis and then we present our approach and finding in later sections. We then conclude the paper with summary of the work and future work plan.

\section{Background}

\section{Mobile Notification}

Notifications are one of the most important features of mobile phones. It is a medium to keep its owner informed and engaged: incoming messages,

engagement on their social media posts, availability of WI-FI networks or applications update, curated nearby places according to their geolocations and e-mail content preview [6]. They come in a form of a brief text and alerting sound (if the speaker is on) or vibrating to catch end-users attention. Notifications being an interruption or multitasking have been a hot topic in Human-Computer Interaction (HCI) community especially in recent years [4]

Once interruptions take place, it is only natural that there will be several effects on people emotion and task performance [1].

Emojis in Mobile Messages

Emojis, which are first found in Japanese mobiles in the ate 1990s, have slowly emerged; starting from Apple's iPhone in 2011 to Android and other operating systems. Current research had indicated that Emojis had already taken over emoticons on social media [10]. This is due

to a richer sets of graphical representation of facial expressions, fun elements for occasions, objects, travels, food and drink, animals, religious, countries and activities. It helps to improve the reader comprehension of the message. With a better representation, Emojis soon become the world fastest growing language; in all form of communications text messages, posts on social media, chatting applications and even in some E-mail applications [3]. Many studies had found that people feel expressing themselves with Emojis is easier than with text. During February, 2014 and August, 2015, about 14\% of public tweets have at least one emoji [11] and half of Instagram text were reported to have Emojis [3]. Not only that Emojis are used in simple communications, but also at corporate level Emojis are utilized in marketing campaign to improve branding and products awareness. Unlike emoticons, emoji cover a wider range of visualization from facial expression to various animals, food activities, objects and weather to cater to different topic in conversation. They were first standardized in Unicode 6.0 and consisted of 722 characters [10]. In 2015, there were approximately 1281 Emojis

characters defined in Unicode 8.0. As the number of Emojis start to grow so as its level of ambiguity and 


\begin{tabular}{|l|l}
\hline $\begin{array}{l}\text { Emoji's Position } \\
\text { in message }\end{array}$ & Occurrences \\
\hline$y n$ & \multirow{2}{*}{ Over $80 \%$} \\
\hline & Less $5 \%$ \\
\hline & About $25 \%$ \\
\hline
\end{tabular}

Table 1: Samples of Emoji's Position in notifications standardization across different platforms, which cause difficulties in sentiment analysis [10].

\section{Sentiment analysis on Emoji}

Being a part of Natural Language Processing (NLP), sentiment analysis has encountered several challenges especially over the sarcasm and ambiguity exist in different natural languages. One of a common NLP approach is to determine the polarity (positive, negative, neutral) of the text, which is achieved by a rule-based methods (lexicon) or machine learning algorithms (Naïve Bayes, Maximum Entropy) [7][11].

Lexicon-based sentiment analysis approaches use sentiment lexicons for retrieving the polarity of individual words and aggregate these scores in order to determine the text's polarity. A sentiment lexicon typically contains simple and compound words and their associated sentiment, possibly differentiated by Part-ofSpeech (POS) and meaning [5]. However, today's lexicon-based approaches typically do not consider emoji. Conversely, one of the first steps in most existing work is to remove many of the Unicode symbols typically constituting Emoji, thus preventing emoji from being detected at all. Yet, state-of-the-art sentiment analysis approaches may be ignoring important information, as an Emoji may for instance articulate emotions when regular text fails to do so e.g., "My day is packed with meetings $\Theta$ ". Therefore, the traditional approach would not be sufficient to analyze the emotional score of emoji. As emoji represent human emotion, according to Paul Ekman's theory, it could be classified as anger, disgust, fearful, happy, sad and surprise [8]. Instagram's engineering team had published their study recently on emoji trends on Instagram. Their study had computed the equivalent English meaning for emoji, which is based on 50 million English comments and captions from Instagram in 2015. Their translation of emoji do not only retrieve a closest meaning, but also semantically retrieve all words that could explain the meaning of emoji in different context. Therefore, it results in a semantic map of Emoji and its context. On the other hand, Kralj Novak et al. aimed to explain emoji from its sentiment point of view [8]. In their study, they had retrieved tweets that contain Emoji in different languages and asked native speakers for respective language to annotate the sentiment of the tweets. The result was a list of emoji and its sentiment ranking based on Lexicon-approach.

In summary, most of the earlier works have focused on Emoji from social media such as Instagram and Twitter. None of them have looked at Emojis in phone notification using real-life data. Such effort will contribute to the ongoing to advance Affect Computing research [9][13].

\section{DATASET and Study Setup}

These notifications are usually appear on the notification panel. NotiMind utilizes the phone's Notification manager API and System Manager API in order to intercept notifications. These notifications are then logged and stamped with time and date of notification activity. In order to ensure privacy compliance, the NotiMind asks for an explicit permission from users to collect to access the user's notifications. Users have to enable this feature directly in the phone settings. Call for participation in the study was advertised in University mailing lists. 12 took part in the study over 5 weeks. For this work, we have looked 
at 446380 notifications from 8 users ( 5 female, 3 male).

\begin{tabular}{|c|c|c|}
\hline Message & $\begin{array}{l}\text { Views is } \\
\text { about to } \\
\text { drop.. } \\
\text { been } \\
\text { waiting } \\
\text { for so } \\
\text { long }(-) \\
(-) \cdot \odot \\
(-)\end{array}$ & $\begin{array}{l}\text { the } \\
\text { features } \\
\text { as well } \\
\text { man } \odot\end{array}$ \\
\hline $\begin{array}{l}\text { Emoji } \\
\text { Count }\end{array}$ & 5 & 1 \\
\hline $\begin{array}{l}\text { Emoji } \\
\text { Description }\end{array}$ & $\begin{array}{l}\text { SMILING } \\
\text { FACE } \\
\text { WITH } \\
\text { HEART- } \\
\text { SHAPED } \\
\text { EYES } \\
\text { SMILING } \\
\text { FACE } \\
\text { WITH... }\end{array}$ & \\
\hline $\begin{array}{l}\text { Text } \\
\text { Sentiment }\end{array}$ & -1 & 0 \\
\hline $\begin{array}{l}\text { Message } \\
\text { Sentiment }\end{array}$ & 19 & 4 \\
\hline
\end{tabular}

Table 2: Sample of notifications data analysis

\section{Data Processing}

An excessive list of cleaning, removing repetitive notification posts, reformatting was required. We used Python as the main programming language to import the SqLite data that sent from Notimind. For this paper, only the description of the notifications is extracted. However, it is also worth noticing the Emojis appearance in message state column, which represents the notification's source. For example, Emoji can appear in a username or a group name. Once the cleaned and formatted, we have extracted Emojis from each message and stored its description. The Emojis were extracted from the notifications retrieved and processed with python script. The sentiment of the notifications will be analyzed using AFINN library for python; the difference between notifications with and without Emojis will be compared along with the Emojis frequency and its position in text.

\section{Discussion}

Type of Emojis and their occurrences:

From our data we found that the number of notifications vary according to the application. There are some Notifications that utilizes an excessive number of Emojis to tell a story or a joke, sometimes referred to as 'CopyPastas'. In this sense, it is hard to interpret and conduct the analysis on them. Regardless, we found 8 commonly used Emoji among all the applications. Most of them are from smiley-face category. As seen in Figure 2, Twitter notifications tend to have more positive looking Emoji whereas Facebook and WhatsApp have a balance use of both positive and negative ones. We also found an interesting correlation between the number of Emojis' occurrence and the sentimental polarity score accumulated. As seen in Figures 3,4,5, the higher the number of Emojis present, the less correlated between the texts sentimental score and the overall message sentimental score. However, with a moderate number of Emojis used will bring a

Emoji's count in notifications from different applications

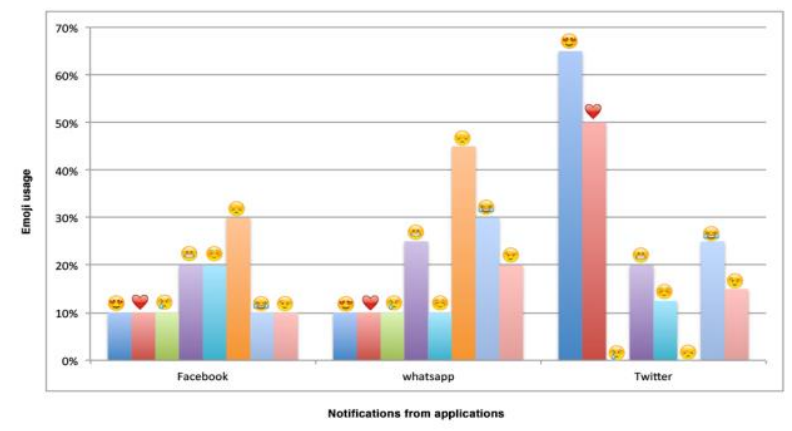

Figure 2: Emojis' count (in percentage) in notifications from Facebook, WhatsApp and Twitter.

higher correlation. It is also worth noticing that in some context, Emoji helps to formulate a more sensible sentiment score than one without see Table 2 . 


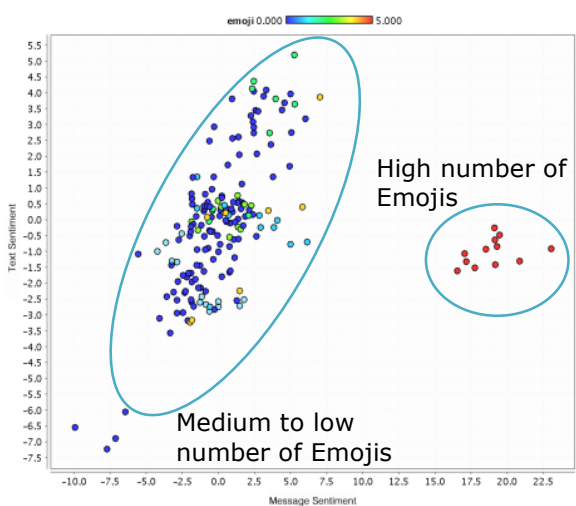

Figure 3: shows correlation between the overall sentimental score from message with Emojis and text retrieved from WhatsApp notifications

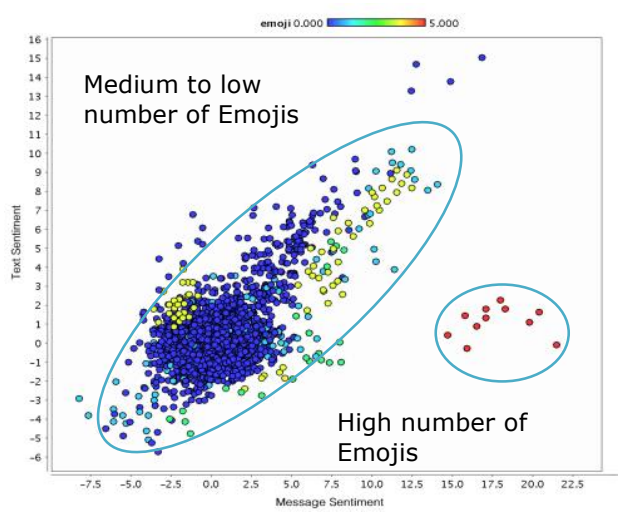

Figure 4: shows correlation between the overall sentimental score from messages with Emojis and text retrieved from Facebook notifications

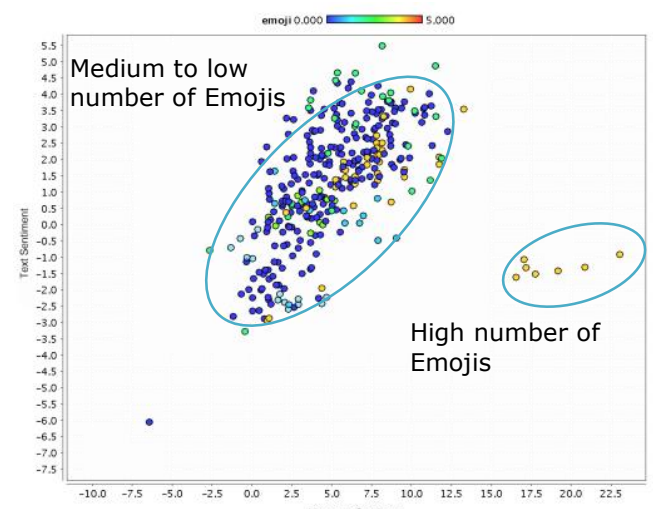

Figure 5: shows correlation between the overal sentimental score from message with Emojis and text retrieved from Twitter notifications
Position of the Emojis: Across the three social network applications, Emojis mainly appear at the end of the message. Only about $25 \%$ of them appear by their own or with one word and less than $5 \%$ appear in the middle of the message. Sample can be found in Table 1.

The majority of notifications received were generated from Facebook (approximately 800 with Emojis).

Twitter and WhatsApp only generated less than half of what Facebook does. The Emojis found in notifications from Facebook covered a bigger group of Emojis than the other two. This is because Facebook offers more interactions. We found that at different context, users tend to use certain category of Emoji. Some of Emojis in Facebook notification that were from status post are from random or decorative Emojis. Those from private messages were from smileys category. Therefore, for notifications that are from messenger type of application, common Emojis were extracted as shown in Figure 2. However, 'love heart ' or "Happyface with love heart eyes:-)" were the most common Emojis in Twitter. Another interesting findings was related to the overall polarity across the three social networks. Unlike Facebook and WhatsApp, Twitter notifications in our data mostly contain positive Emojis. Therefore, the sentiment analysis on Twitter data was shifting towards a positive score. Also, we found that there is a significant relationship between the number of Emojis and the total sentiment score. When there is more than 1 Emoji present in the notification, the pure sentiment of text also correspond with the score obtain from Emojis. However, when the number of Emojis is extremely high, the sentimental score of text and Emoji are no longer correlated. We also found that most Emojis appear at the end of the notification messages. Further inspection has told us that the Emojis that usually appear at the end are more emotionally loader 
Emojis that have a high sentiment score. The common eight Emojis we found in our Findings are from a high sentiment group of Emojis. Other object icons such as " messages or in the $2 / 3$ of the sentence. Their appearance is to help describe the content of the text and not much contribution towards the sentiment.

\section{Conclusion}

In this paper, we investigate the role of Emoji in mobile notifications. Our findings suggest that Emojis often appear in social notifications more than the system notifications. Our initial results imply that Emoji has played a role as an amplifier of the message. Only in some occasion it acts as a modifier of the message. There is a tendency to use more positive Emojis than negative in Twitter, while 'PENSIVE FACE' is more common in WhatsApp and Facebook notifications. The sentimental value of Emojjs is more meaningful when there are multiple Emojis in one notification. In the future, it we will be interested in exploring further the sentimental values of Emojis across more applications by soliciting user's input to label notifications with their emotions in more dimension than positive and

negative. Expressive Emoji could be of sadness, anger, fear, happiness and disgust. As the usage of Emojis grows, it will also be worthwhile to look at the possible evolving of its meaning over time.

\section{References}

1. de Vries, R., Lohse, M., Winterboer, A., Groen, F. and Evers, V. Combining Social Strategies and Workload: a New Design to Reduce the Negative Effects of Task Interruptions. CHI 2013 Extended Abstracts, (2013).

2. Dimson, T. Emojineering Part 1: Machine Learning for Emoji Trends. Instagram Engineering. (2016).

3. Emoji Research Team, Emoji Report. (2015).
4. Gould, S., Cox, A., Brumby, D. and Wickersham, A. Now Check Your Input. Proceedings of the (2016). . CHI Conference on Human Factors in Computing Systems - CHI '16, (2016).

5. Hogenboom, A., Bal, D., Frasincar, F., Bal, M., de Jong, F. and Kaymak, U. Exploiting emoticons in sentiment analysis. Proceedings of the 28th Annual ACM Symposium on Applied Computing, (2013).

6. Iqbal, S. and Horvitz, E. Notifications and awareness. Proceedings of the 2010 ACM conference on Computer supported cooperative work - CSCW '10, (2010).

7. Jurafsky, D. and Martin, J. Speech and language processing. Prentice Hall, Upper Saddle River, N.J., (2000).

8. Kralj Novak, P., Smailović, J., Sluban, B. and Mozetič, I. Sentiment of Emojis. PLOS ONE 10, 12 (2015).

9. Eiman Kanjo, Luluah Al-Husain, and Alan Chamberlain. Emotions in context: examining pervasive affective sensing systems, applications, and analyses. Personal Ubiquitous Computing, 1197-1212, (2015).

10. Miller, H., Thebault-Spieker, J., Chang, S., Terveen, L. and Hecht, B. "Blissfully Happy" or "Ready to Fight": Varying Interpretations in Emoji. the 10th Conference on Web and Social Media, (2016).

11. Pavalanathan, U. and Eisenstein, J. Emoticons vs. Emojis on Twitter: A Causal Inference Approach.CoRR abs/1510.08480, (2015).

12. Nouf Alajmi, Eiman Kanjo, Nour El Mawass, Alan Chamberlain, Shopmobia: An Emotion-Based Shop Rating System. ACII (2013).

13. Luluah Al-Husain, Eiman Kanjo, Alan Chamberlain, Sense of space: mapping physiological emotion response in urban space. UbiComp (Adjunct Publication) (2013). 